ENCYCLOPÉDIE Encyclopédie berbère

BERBERE

18 | 1997

18 | Escargotière - Figuig

\title{
Figue/Figuier
}

(voir A164, Alimentation, Encyclopédie berbère,IV, p. 472-533)

\section{E.B. et S. Chaker}

\section{OpenEdition}

Journals

Édition électronique

URL : https://journals.openedition.org/encyclopedieberbere/2051

DOI : 10.4000/encyclopedieberbere.2051

ISSN : 2262-7197

Éditeur

Peeters Publishers

Édition imprimée

Date de publication : 1 août 1997

Pagination : 2825-2833

ISBN : 2-85744-948-8

ISSN : 1015-7344

Référence électronique

E.B. et S. Chaker, «Figue/Figuier », Encyclopédie berbère [En ligne], 18| 1997, document F23, mis en ligne le 01 juin 2011, consulté le 21 septembre 2021. URL : http://journals.openedition.org/ encyclopedieberbere/2051 ; DOI : https://doi.org/10.4000/encyclopedieberbere.2051

Ce document a été généré automatiquement le 21 septembre 2021.

(c) Tous droits réservés 


\title{
Figue/Figuier
}

(voir A164, Alimentation, Encyclopédie berbère,IV, p. 472-533)

\author{
E.B. et S. Chaker
}

Plus que l'olivier, le figuier est l'arbre par excellence, celui qui est étroitement associé à la vie rurale des Berbères. On le trouve dans tout le Maghreb, d'ouest en est, depuis le Souss et le pays chleuh jusqu'aux confins tripolitains. En latitude, le figuier pousse sur le rivage de la Méditerranée et s'étend jusqu'aux oasis du Sahara central. En altitude, il atteint $1200 \mathrm{~m}$, en Kabylie et dans l'Ouarsenis. C'est donc un arbre ubiquiste mais sa culture n'est pas conduite partout avec le même soin et la production est sujette à de grandes variations. Les principales régions de production sont les Grande et Petite Kabylies, le Tell du nord-est tunisien, le Rif occidental, les vallées de l'Aurès. Dans les ksour de l'Atlas saharien, le figuier est cultivé dans des jardins en concurrence avec l'abricotier et dans la Haute steppe tunisienne, cet arbre fut la seule ressource qui permit aux huit villages accrochés aux montagnes de la Dorsale de résister à la "bédouinisation". Plus au sud, chez les Djebalia du Dahar* et du Demer*, les déplacements de ces semi-nomades sont étroitement liés aux récoltes des trois arbres nourriciers : le figuier, l'olivier et le palmier dattier.

2 Peu exigeant, le figuier pousse dans les fissures des roches aussi bien que dans les bonnes terres, mais si l'arbre est aussi vigoureux sa production est facteur des soins apportés à sa culture et à son entretien. Parmi les régions où le figuier prospère le plus et joue le rôle le plus important dans l'alimentation et la vie sociale, la Grande Kabylie est le pays où sa culture fut la mieux étudiée et à une époque où la région n'avait pas encore subi l'impact de la révolution industrielle. Les premiers travaux, qui sont restés les plus importants, sont ceux de A. Hanoteau et A. Letourneux (1893) dont le lecteur trouvera l'essentiel dans les pages qui suivent; nous avons complété leur texte par des données acquises chez des auteurs plus récents : J. Servier, A. Ibazizen, J.-M. Dallet, G. Laoust-Chantréau. 


\section{Les variétés de figuiers en Kabylie}

3 Le figuier vient partout en Kabylie et on le trouve jusqu'aux altitudes de $1200 \mathrm{~m}$ et, chez les At Ouâban, jusqu'à la zone des cèdres. Les Kabyles distinguent vingt-huit variétés de figuiers outre celles, dont les fruits appelés dukkar servent à la caprification. Les principales variétés sont, parmi les figues blanches: abakur amellal, tayanimt, tazerart, tadefuit... Pour les figues violettes : abakur aberkan, ajanjar, aүanim, tazcišt... La variété appelée tayanimt est la plus estimée parmi les figues blanches et la variété dite ajanjar parmi les figues violettes. On appelle tabuyehbult, une variété de figue plate qui, contrairement aux autres, n'est pas mise à sécher mais consommée toujours fraîche.

\section{Reproduction et culture du figuier}

4 Le figuier se reproduit avec une très grande facilité. Quatre modes de reproduction sont en usage en Kabylie :

1. La bouture immédiate. On casse des branches qui sont déposées pendant quelques jours dans la terre humide, puis on les plante. Les plantations ainsi faites ont l'inconvénient de ne donner des fruits qu'au bout de plusieurs années.

2. Les boutures mises en pépinières. Ces pépinières sont établies dans des terrains irrigables. A la fin de la deuxième ou troisième année, les jeunes plants sont arrachés et transplantés. Ceux qui sont restés trois ans en pépinière produisent dès la deuxième année après la transplantation.

3. Les rejetons pris sur les racines (ušlikan).

4. Les marcottes (urkilen).

5 Lorsqu'on plante un figuier on creuse toujours une tranchée et non un trou rond ou carré comme pour les autres arbres. Le jeune sujet est couché dans cette tranchée et l'on a soin de faire plusieurs incisions sur l'écorce de la partie enterrée, afin de faciliter, pense-t-on, l'émission des racines. Les plantations se font depuis la fin d'octobre jusqu'au commencement de mars. Les figuiers obtenus par ces procédés donnent les mêmes fruits que les arbres dont ils proviennent. Il est inutile de les greffer.

On n'emploie la greffe que pour changer l'espèce ou rajeunir un vieux tronc. Les greffes en usage sont celles pratiquées dans le monde méditerranéen : la greffe en fente sur la tige ou sur la racine, faite en février-mars et la greffe en écusson, en avril-août.

7 Aux approches de l'hiver, tous les bourgeons des figuiers et les extrémités des tiges sont recouverts, par les femmes, d'une couche de bouse de vache destinée à les préserver du gel. Dès les premiers jours de janvier, les travaux commencent dans les vergers de figuiers; ils se continuent ensuite presque sans interruption jusqu'à la récolte. La première opération consiste à déchausser le pied de l'arbre et à établir une cuvette qui retiendra les eaux pluviales. Si la famille dispose de fumier, il sera placé dans cette cuvette. On fait ensuite un premier labour qui sera suivi de trois autres, séparés par un mois d'intervalle. Les époques réputées les plus favorables pour ces labours sont: du 10 au 23 janvier, du 13 au 19 février, du 10 au 22 mars, du 10 au 20 avril et du $1^{\mathrm{er}}$ au 21 mai. Les propriétaires qui n'ont pas de bœufs de labour et sont trop pauvres pour en louer, se contentent de piocher deux ou trois fois le terrain autour des arbres. L'émondage se pratique en hiver, mais l'époque recommandée comme étant de bon augure va du 25 février au 4 mars. 
Les figuiers sont attaqués par une maladie dont les symptômes sont assez semblables à ceux de l'une des maladies du froment pour lui faire donner le même nom. Les Kabyles l'appellent, suivant les localités, maras, kawsses, ou taylalt. Les feuilles jaunissent, se dessèchent et l'arbre meurt, parfois en peu de jours, parfois après une ou deux années de dépérissement. La tailalt apparait surtout dans les bons terrains et s'attaque de préférence aux plus beaux arbres; elle peut dépeupler totalement un verger, les Kabyles attribuent cette maladie à un ver qui rongerait les racines de l'arbre; en fait elle semble plutôt d'origine cryptogamique. Lors du solstice d'été (lkinsla), de grands feux d'herbes humides sont allumés dans les vergers. La fumée qui se répand sur les figuiers chasse la maladie. Un autre moyen de lutter contre elle consiste à suspendre aux branches des crânes d'animaux.

\section{La caprification}

On compte quatre variétés de caprifiguiers ou figuiers mâles dont la tadukkart $n$ tifuzal est réputée la meilleure. Les quatre variétés de dukkar sont employées indistinctement pour tous les figuiers. Aussitôt que les figues commencent à prendre du développement, c'est-à-dire les premiers jours de juin, la caprification débute. Ce sont les femmes qui sont chargées de l'opération qui consiste à suspendre aux branches des figuiers des petits chapelets de trois ou cinq figues mâles. La préparation des dukkar est fort simple : ils sont percés près de la queue avec une aiguille de bois et rassemblés par un brin de jonc ou de toute autre plante; les chapelets ainsi confectionnés sont suspendus aux branches des figuiers, suivant la dimension de l'arbre et la quantité de fruits qu'il porte. Dès que les premiers dukkar commencent à se dessécher et que de nouvelles figues se développent, on pose de nouveaux chapelets. La même opération est ainsi répétée, de quinze jours en quinze jours et jusqu'à six fois dans le courant de l'été.

Quelques tribus, les Ibertrunen, les Maâkta, les At Aïssi*, dont les dukkar sont renommés, en défendent l'exportation hors de leur territoire sous peine de fortes amendes. Mais, en général, à l'époque de la caprification, tous les marchés sont largement approvisionnés de dukkar. Toutes les variétés de figuiers n'ont pas besoin, au même degré, de la caprification.

\section{Les figues fleurs}

11 Plusieurs variétés de figuiers (abakur, taүanimt, ajanjar, azeiš...) produisent deux sortes de fruits : les figues-fleurs, qui croissent à l'aisselle des feuilles inférieures de la branche, et les figues proprement dites, qui se développent sous l'aisselle des feuilles supérieures. Les figues-fleurs mûrissent du commencement de juin au milieu de juillet, c'est-à-dire deux mois environ avant les autres. Elles sont plus grosses mais moins sucrées et sont mangées fraîches.

\section{La récolte des figues}

Quant à la figue ordinaire, elle prend une série de noms suivant son état de développement : chez les At Mangellat (J.-M. Dallet) on l'appelle successivement aqerquš 
(tombée avant maturité), urgel (hâtive), tazubzigt (molle, presque mûre), amellaggu (figue qui commence à sécher et dont la chair devient comme une pâte confite).

Dès que les premières figues grossissent on entame, chez les Kabyles, une période de quarante jours appelée tesentit, pendant laquelle il est interdit de cueillir les fruits. Cet interdit est le haq $n$-tbexsisin; il s'applique aux figues qui sont sur l'arbre et non sur les fruits tombés à terre. En cas d'infraction à cette règle on paye une amende. Antérieurement, cet interdit collectif était accompagné d'une malédiction, le $d \varepsilon \hat{a}$, qui était prononcée solennellement par un marabout, lors d'une assemblée du village convoquée par l'amin. L'anathème était prononcé contre ceux qui ne respecteraient pas les décisions de l'assemblée, contre ceux qui porteraient la main sur les figues mûrissantes et qui les consommeraient clandestinement. Ces fruits se transformeront en oiseaux noirs qui picoreront de leur bec l'estomac des contrevenants.

14 Si un propriétaire de figuiers particulièrement réputés juge insuffisant le poids de la malédiction, il peut assurer lui-même la garde de ses fruits pendant la période de mûrissement; pour cela il construit, dans l'arbre le plus élevé du verger une sorte de guérite en branchage, la siwan où il passe la nuit (A. Ibazizen).

Lampe au gardien ( $v^{\mathrm{e}}$ siècle de notre ère).

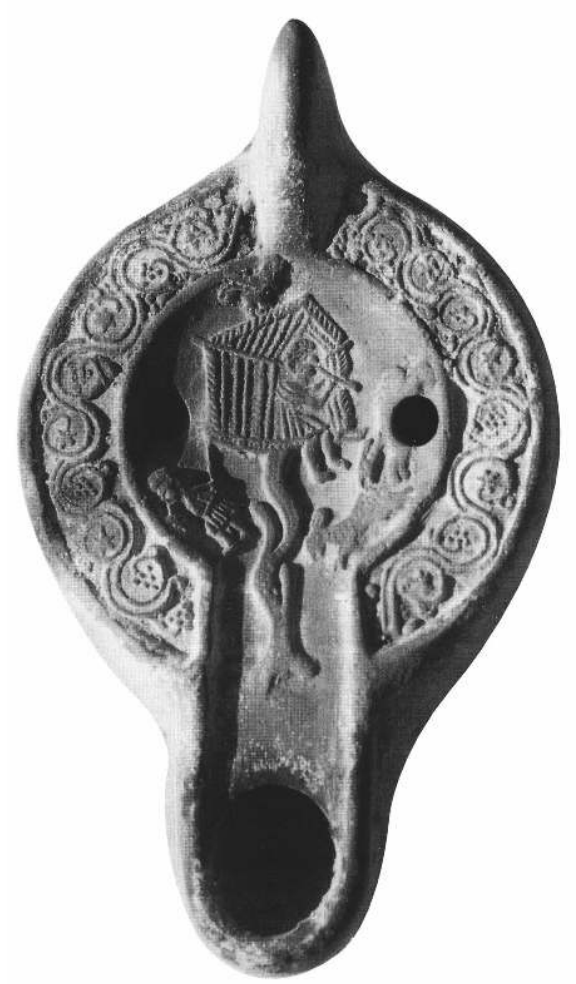

Dans sa cabane de surveillance, le gardien joue de la flûte après avoir capturé un maraudeur qu'il a suspendu par la ceinture au plancher de son abri. Le gardien est généralement considéré comme surveillant de la vigne, mais, comme dans la Kabylie actuelle, cette surveillance peut s'étendre à la production de figues.

Certaines lampes romaines d'Afrique représentaient déjà un gardien juché dans de telles cabanes. 

consommation des figues peut commencer. Elles étaient attendues avec d'autant plus d'impatience que ce début du mois d'août faisait suite à une période de privation et que chaque famille risquait de succomber à la tentation de consommer, jour après jour, les excellents fruits au lieu de tenter de les conserver pour les consommer tout au long de l'année.

17 Cette conservation dépend de la dessiccation des figues. Dans chaque jardin, les paysans, en fait surtout leurs femmes, font sécher la récolte de figues au grand soleil sur des claies de roseaux ou d'asphodèles. Chaque soir ces claies sont superposées pour les protéger de l'humidité ; la claie supérieure recevra une couverture de plaques de lièges et de pierres plates. Mieux protégées que les autres et reconnues pour leur délicatesse, les figues de cette claie ont été choisie une à une. Les propriétaires de plusieurs figuiers, dont la récolte est généralement importante, réserve un local, hutte de branchage ou cabane de pierres pour protéger leurs figues de l'humidité durant la nuit. entassent dans un akufi* après en avoir tapissé le fond de feuilles de figuier. Quand l'énorme akufi est à demi rempli, on y fait entrer un enfant qui tasse les figues avec les pieds. Dans les régions basses de la Kabylie on prend soin d'arroser d'eau salée parfumée au laurier les figues entassées pour les protéger des parasites. Dans les villages du Djurdjura le climat rigoureux suffit à assurer une conservation satisfaisante (G. Laoust-Chantréaux, p. 95-96). Chez les tribus du bord de la mer (At Waguennun, Iflissen*, At Djennad, Izerxfawen) les figues sont conservées d'une manière différente : elles sont au préalable, ouvertes et aplaties, puis après la dessiccation, elles sont placées par couches successives dans de grands moules de bois où elles sont tassées au moyen d'une presse à vis. L'opération terminée, les figues forment un gros pain auquel on donne le nom de tifrešt et qui fait l'objet d'une exportation.

\section{Importance de la figue dans l'alimentation}

La figue joue un grand rôle dans l'alimentation des Kabyles; elle est consommée de deux manières, à l'état frais (elle est dite alors lexrif) et surtout, durant tout le reste de l'année, sous forme séchée. La figue sèche est appelée dans tout l'ensemble berbérophone tazart (tahart en tamahaq), ce nom a fini par désigner aussi bien l'arbre que le fruit en tamazirt du Maroc central, et en tamahaq de l'Ahaggar.

Les Kabyles mangent des quantités considérables de figues fraîches après la levée de l'interdit et durant la récolte et la dessiccation des fruits. L'abondance de sucre absorbé n'est pas sans rapport avec une espèce d'ivresse qui gagne la collectivité et se manifeste par nombre de querelles, voire de combats de rue entre les jeunes appartenant à des çofs* opposés. Aussi, concluent A. Hanoteau et A. Letourneux, «la saison des figues est toujours une époque de rixes et de batailles ».

21 Mais cette période, appelée comme la figue fraîche : lexrif était ressentie comme particulièrement faste. Comme l'écrit A. Ibazizen : «Bien avant l'institution sociale des congés payés, cette période était choisie de préférence à toute autre par les natifs du pays pour se retremper dans leur milieu. Après le temps de pauvreté, sinon de misère,

Encyclopédie berbère, 18 | 1997 
que vivait la Kabylie en hiver et au printemps il lui venait trois mois d'une abondance relative sous le règne du figuier. »

Maison de Kabylie maritime et son verger de figuiers en hiver.

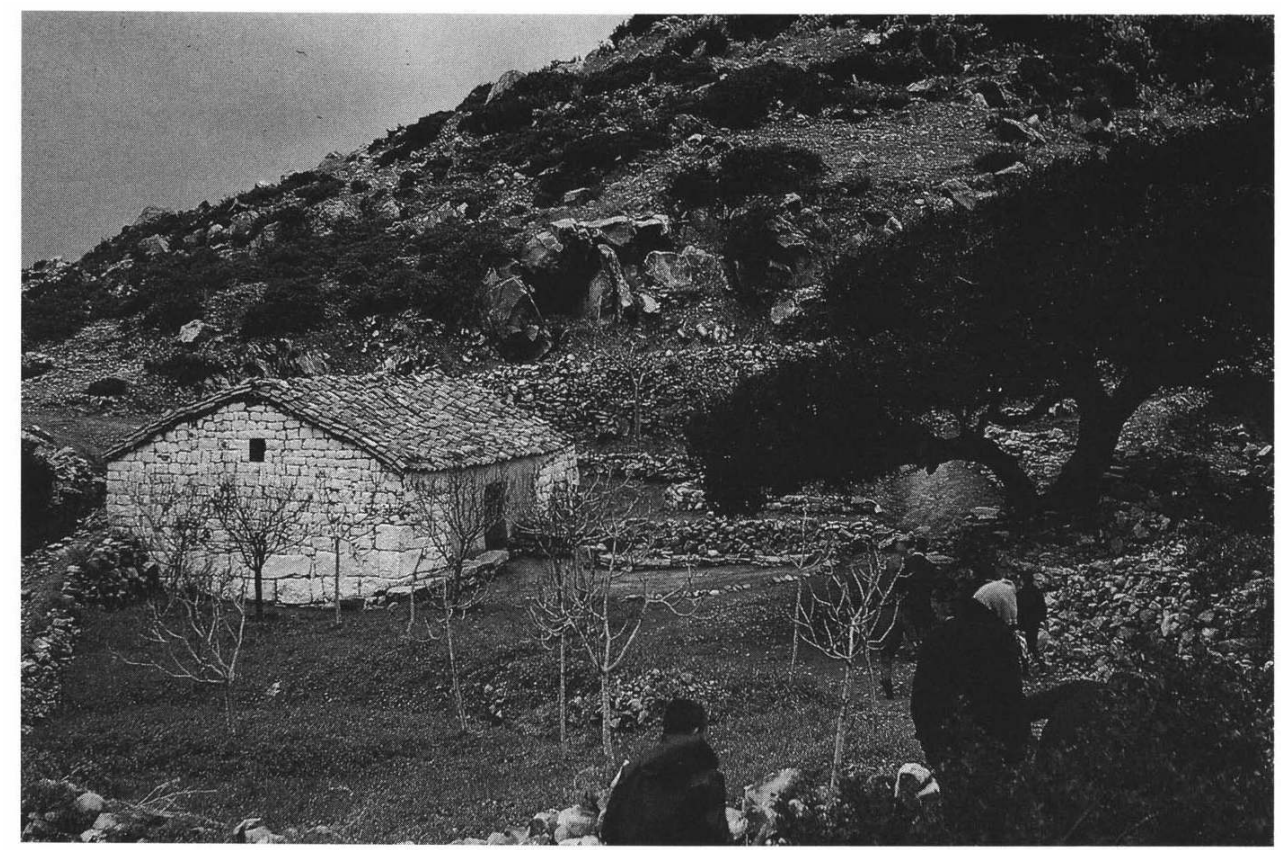

Photo G. Camps.

Pendant le reste de l'année, la figue sèche constitue l'essentiel de la nourriture. Deux repas sur quatre sont composés de tazart (figue sèche). Tous les repas pris en dehors de la maison sont à base de figues sèches trempées ou non dans l'huile d'olive. Le colporteur kabyle, qui était une image familière de l'Algérie du XIXe siècle, n'emportait, pour se rendre à Alger et en revenir, que deux ou trois galettes et un kilogramme de figues sèches.

\section{Rôle de la figue dans les pratiques agraires traditionnelles}

Dans tous les pays Berbères du Maghreb la figue est un élément très important de la magie propitiatoire, particulièrement dans les rites agraires, mais aussi dans les pratiques familiales liées à la fécondité. Partout la figue par la profusion de ses graines se trouve en concurrence avec la grenade, autre image de la fécondité en raison de ses nombreux grains ; aussi très souvent ces deux fruits sont ils associés dans les offrandes. C'est le cas, en particulier, dans les rites de labour où la pratique la plus courante consiste, pour la femme arrivée à la limite du champ à labourer, à tendre à son mari figues et grenades, que celui-ci enterre au début du premier sillon. D'après J. Servier, pour les paysans du Chenoua*, cette offrande déposée dans le premier sillon a un sens très précis et détaillé : les figues sèches représentent le blé, les grenades, l'orge et parfois on complète l'offrande par des caroubes qui doivent assurer une bonne récolte de fèves. 
Mais les figues, fraîches ou sèches, ont d'autres qualités et pouvoirs. Chez les At Djennad, les repas communiels, qui sanctionnent un événement important, sont frugalement constitués de pain levé et de figues. C'est encore en raison de leurs promesses de fécondité que des figues sèches placées dans un tamis sont, dans l'Aurès, offertes par sa belle-mère à la jeune mariée, lors de son arrivée au domicile conjugal.

\section{FIGUIER/FIGUE(S) (nom berbère [azar/tazart]) (S. Chaker)}

Le terme berbère fondamental désignant la figue (ou le figuier) en tant que ressource alimentaire de base est remarquablement stable à travers l'ensemble du domaine.

Bien sûr, comme le montre la contribution générale sur le figuier (cf. supra) il existe, dans les régions qui pratiquent intensivement la culture du figuier, un foisonnement de désignations, propres à chaque variété de figue, en fonction de la couleur, de la taille, du calendrier et de l'état de maturation, des caractéristiques gustatives... Sur ce champ lexical, on dispose d'une étude précise consacrée au domaine kabyle : Haddadou 1985. Indépendamment des dénominations de variétés particulières, on considérera aussi comme secondaires des formes comme le mozabite (Delheure 1984 p. 124) : amešši/ tameššit "figue", ou le chaouïa (Huyghe 1906, p. 287) : tameččit/imečči "figue verte". Cette appellation procède manifestement de la spécialisation d'un terme au départ très général, issu de la racine ešš/ečč manger ( = "ce qui se mange, nourriture").

Mais il existe bien une dénomination générale, commune quasiment à l'ensemble des dialectes berbères, du Maroc à la Kabylie, des Aurès au monde touareg : azar, tazart

- Kabyle (Dallet 1982 : 954) : tazart, "figues sèches".

Il s'agit ici d'un collectif, s'appliquant aux figues sèches en tant qu'aliment de base (au même titre que le blé et l'huile d'olive). Le caractère "basique" en est souligné par l'absence de toute forme de pluriel : les figues sèches sont posées comme un ensemble non dénombrable et non segmentable, une "matière première", en somme; il existe d'ailleurs dans ce dialecte un verbe spécial "acheter/commercer", s'appliquant exclusivement aux figues sèches, aux céréales et à l'huiler : ağew, qui marque bien la spécificité de ces produits dans le système économique et alimentaire de la société kabyle traditionnelle.

- Chleuh (Destaing 1938 : 128) : tazart, "figue" (collectif et nom d'unité) ; addag $n$ tazart " figuier" ( $=$ arbre de figues).

- Mzab (Delheure 1984 : 254) : azar/azaren et tzart/tizarin, "baie, jujubes".

- Ghadames (Landry 1973 : 429, № 1829) :

azar/azaren "fleur et fruit du jujubier sauvage".

tazart/tazarin "figue sèche"

- Chaoui (Huyghe 1906 : 287) :

tazart (collectif) "figuier"

- Rif (Renisio $1932:$ 323, 423) :

tazart, "figue"

zara, "verger" et "jardin de figuiers"

- Tamazight (Mercier 1937 : 117-118 \& Taïfi 1991 : 812) : tazart/tazarin, "figue"; aseklu $n$ tazart, "figuier" ( = arbre de figues). 
La forme azar/tazart est donc remarquablement stable et appartient incontestablement au fond lexical berbère commun, du fait de sa très large distribution. Son signifié est un peu moins bien défini, mais il désigne, presque partout, de manière générique, "la figue". En deux points (Mzab et Ghadames), le terme s'applique aussi à la baie de jujubier sauvage. Deux hypothèse explicatives peuvent être envisagées :

- ou bien, il s'agit d'une évolution sémantique locale, un déplacement du réfèrent lié à la rareté du figuier dans les deux régions concernées ;

- ou bien - et l'on penchera plutôt pour cette explication -, on a affaire à la conservation partielle d'un signifié antérieur, qui pouvait être: "fruit, baie (générique)", à partir duquel ont émergé des significations plus précises, avec une certaine marge de variation liée aux conditions climatiques: le "fruit/la baie par excellence" n'est évidemment pas la même en Kabylie et au Sahara...

Que la signification "baie/jujube sauvage" soit attestée en deux endroits au moins n'est probablement pas fortuit et pourrait impliquer que la base lexicale azar/tazart ait désigné au départ, tout fruit ou baie sauvage, antérieurement même à l'apparition de l'agriculture. On sait notamment que les jujubes ont constitué un élément important de l'alimentation locale, depuis les temps préhistoriques.

En tout état de cause, on peut considérer comme acquis que le terme azar/tazart :

- est très ancien puisque pan-berbère ;

- qu'il a désigné le "fruit (consommable) par excellence";

- que sa signification "figue/figuier" est très anciennement constituée, non seulement en raison de sa très large distribution, mais aussi du fait qu'elle est attestée dans des zones sahariennes où le figuier est rarissime (touareg de l'Ahaggar).

Enfin, si l'hypothèse paléo-linguistique d'un signifié primitif "fruit/baie (sauvage) " est exacte, ce lexème azar/tazart pourrait être rapproché de différentes formes issues de racines $Z R, Z(W) R$ et $Z(W) R$ (avec emphatisation du /z/) qui désignent, à travers tout le monde berbère, divers fruits de forme ronde: tiẓurin "raisins", azegg ar (< ZWR) " jujubier"... Cf. Laoust 1920 : 421-422.

3 Plus largement, il n'est pas exclu que cette base lexicale ait un lien avec diverses formes lexicales proches, autour de la séquence $Z R(R)$, qui évoquent les notions de "grain, gravillon" et "collier (de perles)" : tazra, azrar/tazrart... (kabyle, Ouargla, tamazight, chleuh...).

34 On sera alors tenté de rapprocher les formes berbères de la racine sémitique et chamito-sémitique $Z R \mathcal{E}$, "graine, semence...", avec chute, normale en berbère, de la pharyngale sonore finale et compensation par la stabilisation et l'allongement de la voyelle ouverte médiane / $\mathrm{a} /$ (thème nominal zar), pratiquement présente dans les toutes variantes relevées du lexème azar/tazart. Dans cette constellation lexicosémantique, le lexème pan-berbère tamazirt, "jardin", "verger", "pays", pourrait bien être un participe passif issu d'une la racine ${ }^{*} Z R>$ mazir "ce qui est ensemencé, cultivé"?).

On perçoit donc assez nettement l'existence en berbère d'une "proto-racine" $Z R$ qui renvoie aux notions de "graine, grain rond, fruit (rond)".

En définitive, pour ce qui est du berbère azar/tazart "figue", tout confirme ce qu'écrivait, dès 1961, Gabriel Camps qui avait parfaitement mesuré l'intérêt et les implications des données linguistiques : 


\section{BIBLIOGRAPHIE}

HANOTEAU A. et LETOURNeUX A., La kabylie et les coutumes kabyles, Paris, Challamel, 1893

IBAZIZEN A., Le pont de Berq'mouch. La Table ronde, 1979

DALLET J.-M., Dictionnaire kabyle-français. Parler des At Mangellat. Paris, Selaf, 1982

SERVIER J., Traditions et civilisation berbères. Monaco, éditions du Rocher, 1985

LAOUST-CHANTRÉAUX G., Kabylie côté femmes. La vie féminine à Ait Hichem. Aix-en-Provence, Édisud, 1990.

«La figue serait donc le fruit par excellence, celui qui prévaut sur tous les autres, ce qui est conforme à la place prépondérante que tient te fruit dans l'alimentation de nombreux montagnards » $(1961: 90)$.

CAMPS G, Massinissa ou les débuts de l'Histoire, Alger, 1961.

CORTADE J.-M., Lexique français-touareg (dialecte de l'Ahaggar), Paris, 1967.

DALLET J.-M., Dictionnaire kabyle-français, Paris, 1982.

DELHEURE J., Dictionnaire mozabite-français, Paris, 1984.

DELHEUREJ., Dictionnaire ouargli-français, Paris, 1987.

Destaing E., Vocabulaire français-berbère (tachelhit du Souss), Paris, 1938.

FOUCAULD CH. DE, Dictionnaire touareg-français, Paris, 4 vol. , 195052.

HADDADOU M., Structures lexicales et significations en berbère (kabyle), Thèse de doctorat de $3^{\mathrm{e}}$ cycle, Université de Provence, 1985, p. 304-314 : “Le vocabulaire kabyle de la figue”.

HUYGUE G., Dictionnaire français-chaouia, Alger, 1906.

LANFRYJ., Ghadames II (Glossaire), Alger, FDB, 1973.

LAOUST E., Mots et choses berbères, 1920 (1983).

MERCIER A., Vocabulaire et textes berbères dans le dialecte des Aït Izdeg, Rabat, 1937.

RENISIO A., Étude sur les dialectes berbères des Beni Iznassen, du Rif..., Paris, 1932.

TAÏFI M., Dictionnaire tamazight-français (Parlers du Maroc central), Paris, L’Harmattan-Awal, 1991.

\section{INDEX}

Mots-clés : Agriculture, Algérie 